divider," IEEE Trans. Microwave Theory Tech., vol. MTT-17, pp. 406-408, July 1969.

[7] S. B. Cohn, "A class of broadband three-port TEM-mode hybrids," IEEE Trans. Microwave Theory Tech., vol. MTT-16, pp. 110-116, Feb. 1968.

[8] H. Y. Yee, F.-C. Chang, and N. F. Audeh, " $N$-way TEM-mode broad-band power dividers," IEEE Trans. Microwave Theory Tech., vol. MTT-18, pp. 682-688, Oct. 1970.

[9] J. R. Dent, "Strip-line technique produces a simple 3-dB directional coupler," Electronic Design, pp. 52-53, Aug. 31, 1960.

[10] J. T. Bangert, R. S. Engelbrecht, E. T. Harkless, R. V. Sperry, and E. J. Walsh, “The spacecraft antennas," Bell Syst. Tech. J., vol. 42, no. 4, part 1, pp. 869-897 (figs. 8,9), July 1963.

[11] H. Fukui, "Frequency locking and modulation of microwave silicon avalanche diode oscillators," Proc. IEEE (Lett.), vol. 54, no. 10, pp. 1475-1477, Oct. 1966.

[12] P. C. Goodman, "A wideband stripline matched power divider," in 1968 Int. Microwave Symp. Dig. Tech. Papers (IEEE Cat. No. 68C38), pp. 16-20, May 1968.

[13] J. Hughes and K. Wilson, "High power multiple IMPATT amplifiers," in Conference Proceedings of the 4th European Microwave Conference. Kent, England: Microwave Exhibition and Publishers Ltd., Sept. 1974, pp. 118-122.

[14] U. H. Gysel, "A new $N$-way power divider/combiner suitable for high-power applications" 1975 IEEE MTT-S Int. Microwave Symp. Dig. (IEEE Cat. No. 75CH0955-5MTT), pp. 116-118, May 1975.

[15] H. C. Chappell, "Designing impedance matched in-phase power dividers," Microwave J., vol. 22, no. 2, pp. 51-52, Feb. 1979.

[16] L. I. Parad and R. L. Moynihan, "Split-tee power divider," IEEE Trans. Microwave Theony Tech., vol. MTT-13, pp. 91-95, Jan. 1965.
[17] R. B. Ekinge, "A new method of synthesizing matched broad-band TEM mode three ports," IEEE Trans. Microwave Theory Tech. vol. MTT-19, pp. 81-88, Jan. 1971.

[18] R. P. Tetarenko and P. A. Goud, "Broad-band properties of a class of TEM-mode hybrids," IEEE Trans. Microwave Theory Tech., (Corresp.), vol. MTT-19, pp. 887-889, Nov. 1971.

[19] H. Kagan, "N-way power divider," IRE Trans. Microwave Theory Tech. (Corresp.), vol. MTT-9, pp. 198-199, Mar. 1961.

[20] S. V. Bearse, Ed., "Compact radial power combiner teams up a dozen power GaAs FETs," Microwaves, vol. 16, no. 10, p. 9, Oct. 1977.

[21] J. M. Schellenberg and M. Cohn, "A wideband radial power combiner for FET amplifiers," in 1978 IEEE Int. Solid-State Circuit Conf. Dig. (IEEE Cat. No. 78CH1298-9SSC), pp. 164-165, 273, Feb., 1978.

[22] M. Cohn, B. D. Geller, and J. M. Schellenberg, "A 10-watt broadband FET combiner/amplifier," in 1979 IEEE MTT-S Int. Microwave Symp. Dig. (IEEE Cat. No. 79CH1439-9MTT), pp. 292-297, Apr. 1979.

[23] Z. Galani and S. J. Temple, "A broadband $N$-way combiner/divider," in 1977 IEEE MTT-S Int. Microwave Symp. Dig. (IEEE Cat. No. 77CH1219-5MTT), pp. 499-502, June 1977.

[24] "X-band planar combiner module using GaAs FETs," Microwave J., vol. 21, no. 2, p. 34, Feb. 1978.

[25] N. Nagai, E. Maekawa, and K. Ono, "New $n$-way hybrid power dividers," in 1977 IEEE MTT-S Int. Microwave Symp. Dig. (IEEE Cat. No. 77CH1219-5MTT), pp. 503-504, June 1977.

[26] C. G. Montgomery, R. H. Dicke, and E. M. Purcell, Principles of Microwave Circuits (M.I.T. Rad. Lab. Series, Vol 8). New York: McGraw-Hill, 1948, eq. (87), p. 148.

[27] A. A. M. Saleh, Bell Syst. Tech. J., to be published.

\title{
Reflection Coefficient Transformations for Phase-Shift Circuits
}

\author{
HARRY A. ATWATER, SENIOR MEMBER, IEEE
}

\begin{abstract}
It is shown how switchable one-port circuits having two impedance states may be transformed so as to exhibit reflection coefficients which have a prescribed phase angle difference and equal magnitude in the two states. In reflection-type phase shifters, arbitrary phase shift may be obtained without change of signal amplitude. The reflection properties are achieved by the use of an impedance-transforming two-port network. Design equations and an example are given.
\end{abstract}

\section{INTRODUCTION}

I N THE DESIGN of phase-shift circuits for applications in phased array steering and phase modulators, switching circuits based on solid-state components have advantages of compact size and high switching speed. Semiconductor-based phase shifters also have the poten-

Manuscript received August 24, 1979; revised December 7, 1979. This work was supported by the U.S. Department of the Navy.

The author is with the Lincoln Laboratory, Massachusetts Institute of Technology, Lexington, MA 02173. tial for integration on a single chip with their associated circuits. In digitally controlled phase shifters, phase-shift elements are required which ideally change the phase of the transmitted signal by a specified amount without altering its amplitude. In the usual formulation of these circuits, two-port elements in cascade introduce a discrete step or bit in phase of the transmitted signal. Circuit forms which have been employed are: path-switching circuits, loaded-line phase shifters, and reflection-type phase shifters used in conjunction with a hybrid coupler. Phase-shift circuit technology has been extensively reviewed [1]-[4].

\section{Reflection-Type Phase Shifters}

The reflection-type circuit has been employed in several recent phase shifter designs [5]-[7]. The basic reflectiontype phase shifter consists typically of switchable imped- 


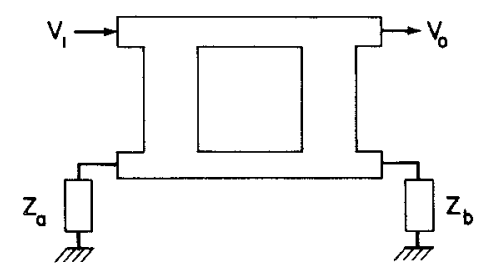

(a)

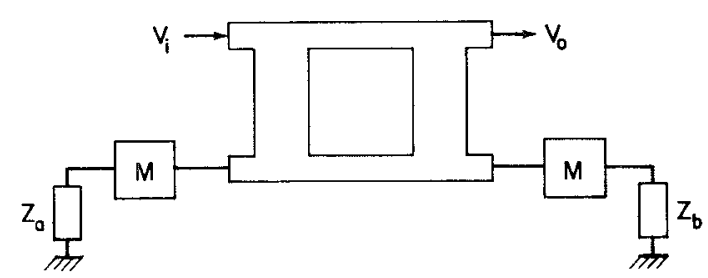

(b)

Fig. 1. (a) Terminated hybrid coupler for reflection phase shifter. (b) Coupler with transformed terminating impedances.

ance elements $Z_{a}$ and $Z_{b}$ terminating two ports of a 3-dB hybrid tee, with the output of the tee being taken from the normally decoupled port (Fig. 1(a)). The output signal from the hybrid is

$$
V_{0}=j \frac{V_{i}}{2}\left[\Gamma_{a}\left(Z_{a}\right)+\Gamma_{b}\left(Z_{b}\right)\right]
$$

where $V_{i}$ is the ingoing wave, and $\Gamma_{a}\left(Z_{a}\right)$ and $\Gamma_{b}\left(Z_{b}\right)$ are the reflection coefficients of terminations $Z_{a}$ and $Z_{b}$. If $Z_{a}$ and $Z_{b}$ are equal, the reflected wave at the input is zero. It will be assumed in the following that $Z_{a}=Z_{b}=Z$. It is assumed that impedances $Z$ contain a semiconductor element which may be biased to its "off" or "on" state, producing input impedances $Z_{1}$ and $Z_{2}$, respectively. The output of the phase shifter will have the desired characteristic of constant signal amplitude with phase angle change $\phi$ if

$$
\Gamma_{1}\left(Z_{1}\right)=e^{j \phi} \Gamma_{2}\left(Z_{2}\right)
$$

The condition of (1) will not normally be true for terminating impedances consisting of a $\mathrm{p}-\mathrm{i}-\mathrm{n}$ diode or similar semiconductor element. In principle, $180^{\circ}$ phase shift with unit reflection amplitude is achieved by switching between an ideal short circuit and an ideal open circuit. However, for a termination consisting of $p-i-n$ diode having a forward resistance of $3 \Omega$ and a capacitance of $0.05 \mathrm{pF}$ under reverse bias, a phase shift of only about $160^{\circ}$ is possible in the reflected wave at $10 \mathrm{GHz}$. A section of short-circuited transmission line may be connected in shunt with the diode in order to increase the amount of phase shift available, or other tuning elements and circuit modifications may be introduced in order to adjust the amount of phase shift or improve the uniformity of the reflected signal in amplitude. These modifications may be arrived at empirically or by trial and error methods [8], [9]. It is shown in the following sections, however, how an impedance-transforming network may be designed for a given two-state termination or one-port element which yields a prescribed change in the phase
TABLE I

\begin{tabular}{l}
\hline \hline$\hat{\beta}_{\Gamma} \rightarrow\left(\begin{array}{cc}1 & -Z_{0} \\
1 & Z_{0}\end{array}\right)$ \\
$\hat{\beta}_{M} \rightarrow\left(\begin{array}{ll}A & B \\
C & D\end{array}\right)$ \\
$\hat{\beta}_{\phi} \rightarrow\left(\begin{array}{ll}e^{j \phi / 2} & 0 \\
0 & e^{-j \phi / 2}\end{array}\right)$ \\
\hline
\end{tabular}

angle of the reflection coefficient $\Gamma$ upon switching, while maintaining constancy of the magnitude of $\Gamma$.

\section{Derivation of Transformation Equations}

The impedance transformation by a two-port is

$$
Z_{n}^{\prime}=\frac{A Z_{n}+B}{C Z_{n}+D}
$$

where $n=1,2$, correspond to impedance states before and after switching respectively, and $A, B, C, D$ are the elements of the matrix $(M)$ of general circuit parameters. The reflection coefficients of the transformed impedance states are

$$
\Gamma_{n}^{\prime}\left(Z_{n}^{\prime}\right)=\frac{Z_{n}^{\prime}-Z_{0}}{Z_{n}^{\prime}+Z_{0}}, \quad n=1,2
$$

and (1) assumes the form

$$
\Gamma_{1}^{\prime}\left(Z_{1}^{\prime}\right)=e^{j \phi} \Gamma_{2}^{\prime}\left(Z_{2}^{\prime}\right)
$$

Equations (2) and (3) have the form of bilinear transformations, or fractional linear transformations [10]. The successive application of two bilinear transformations is a bilinear transformation. The symbol $\hat{\beta}_{\Gamma} Z_{n}^{\prime}$ will be used here to represent the application of the bilinear transformation of (3) to $Z_{n}^{\prime}$. Similarly, using $\hat{\beta}_{M} Z_{n}$ to represent the right side of (2), (4) may be expressed in the form

$$
\hat{\beta}_{\Gamma} \hat{\beta}_{M} Z_{1}=e^{j \phi} \hat{\beta}_{\Gamma} \hat{\beta}_{M} Z_{2}
$$

A simple product of two factors may be represented by a bilinear transformation. Multiplication of function $f$ by the factor $e^{j \phi}$ may be represented by the transformation

$$
\hat{\beta}_{\phi} f=\frac{e^{j \phi / 2} f+0}{0+e^{-j \phi / 2}} .
$$

Equation (5) then becomes

$$
\hat{\beta}_{\Gamma} \hat{\beta}_{M} Z_{1}=\hat{\beta}_{\phi} \hat{\beta}_{\Gamma} \hat{\beta}_{M} Z_{2}
$$

There is a group correspondence between the application of bilinear transformations and the multiplication of $2 \times 2$ matrices (10),(11). Matrices corresponding to the transformations which have thus far been introduced are given in Table $I$.

The inverse bilinear transformation, corresponding to the removal of a specific transformation, has as its matrix counterpart the inverse of the transformation matrix. Representing the inverse of transformation $\hat{\beta}_{x}$ by the symbol $\hat{\beta}_{x}^{-1}$, operating on (7) from the left with the transformation $\hat{\beta}_{M}^{-1} \hat{\beta}_{\Gamma}^{-1}$ yields the following result:

$$
Z_{1}=\hat{\beta}_{M}^{-1} \hat{\beta}_{\Gamma}^{-1} \hat{\beta}_{\phi} \hat{\beta}_{\Gamma} \hat{\beta}_{M} Z_{2} \equiv \hat{\beta}_{B} Z_{2}
$$




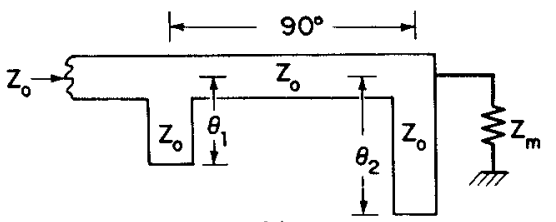

(a)

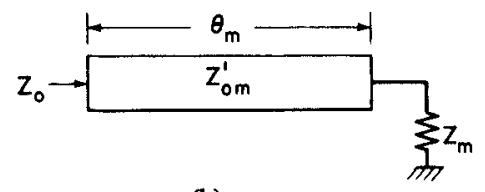

(b)

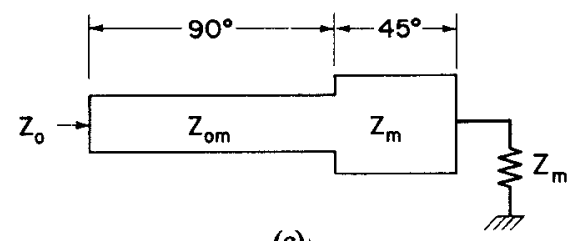

(c)।

Fig. 2. Two-port impedance transformers. (a) Double-stub transformer with quarter-wavelength spacing. (b) Line segment of characteristic impedance $Z_{0 m}^{\prime}$ and electrical length $\theta_{m}$. (c) Line segment of electrical length $90^{\circ}$ and characteristic impedance $Z_{0 m}$ in tandem with $45^{\circ}$ segment of characteristic impedance $Z_{m}$

The combined transformation $\hat{\beta}_{B}$ represents a mapping which maps the final-state impedance $Z_{2}$ onto its initial state $Z_{1}$. This overall transformation is represented by the matrix product

$$
\begin{aligned}
\left(\begin{array}{ll}
B_{11} & B_{12} \\
B_{21} & B_{22}
\end{array}\right) & =\left(\begin{array}{rr}
D & -B \\
-C & A
\end{array}\right)\left(\begin{array}{cc}
1 / 2 & 1 / 2 \\
-Y_{0} / 2 & Y_{0} / 2
\end{array}\right) \\
& \cdot\left(\begin{array}{ll}
e^{j \phi / 2} & 0 \\
0 & e^{-j \phi / 2}
\end{array}\right)\left(\begin{array}{rr}
1 & -Z_{0} \\
1 & Z_{0}
\end{array}\right)\left(\begin{array}{ll}
A & B \\
C & D
\end{array}\right)
\end{aligned}
$$

where $Y_{0}=1 / Z_{0}$.

Using the combined matrix $B_{i j}$ defined by (9), equation (8) is equivalent to

$$
B_{21} Z_{1} Z_{2}+B_{22} Z_{1}-B_{11} Z_{2}-B_{12}=0
$$

Equation (10) represents the basis for the design of the required impedance transforming network. In general, each factor $B_{i j}$ depends on all four elements $A, B, C, D$ of the two-port impedance transforming network. When the real and imaginary parts of (10) are separated, the resulting two equations are not sufficient to determine the four unknowns $A, B, C, D$. Therefore, it becomes necessary to define the coupling network in terms of two parameters. A variety of available matching circuits may be specified in terms of two parameters. These include the double stub impedance transformer with quarter-wavelength spacing, the two stub lengths $\theta_{1}$ and $\theta_{2}$ being the free variables; a line segment of characteristic impedance $Z_{0 m}^{\prime}$ and length $\theta_{m}$; or also a pair of line segments of fixed length in tandem, with their characteristic impedances $Z_{0 m}$ and $Z_{m}$ being the free parameters (Fig. 2). Using any of these impedance transforming networks, (10) may be solved for the design values of the parameters in terms of the known terminating-impedance values $Z_{1}$ and $Z_{2}$.

A more general method of description of a two-port matching network is available however in terms of its "matching impedance" $Z_{m}$, which is defined as that impedance which would be transformed to the input line characteristic impedance $Z_{0}$ by the network. This is summarized by the requirement

$$
Z_{0}=\frac{A Z_{m}+B}{C Z_{m}+D}=\hat{\beta}_{M} Z_{m}
$$

Since the virtual impedance $Z_{m}$ is matched to $Z_{0}$ by the matching network, its reflection coefficient vanishes. The bilinear operator expression for its condition is

$$
\hat{\beta}_{\Gamma} \hat{\beta}_{M} Z_{m}=0
$$

By use of (12) in (5), it is possible to determine $R_{m}$ and $X_{m}$, the real and imaginary parts of $Z_{m}$, in terms of the known values $Z_{1}$ and $Z_{2}$ of the two-state impedance being transformed and the angle $\phi$ of phase shift. Details of this calculation and the resulting expressions for $R_{m}$ and $X_{m}$ are given in the Appendix.

The introduction of bilinear notation is not essential to the determination of the reflection coefficient transforming network. Equation (4) may of course be solved by conventional algebraic means. The use of the bilinear forms serves to display the structure of the problem and to organize its solution. The design of reflection coefficient transforming networks has previously been discussed by Navarro [12] and Steinbrecher [13]. Steinbrecher presented design equations which are in agreement with those obtained analytically here, but obtained his results by means of a construction based on the hyperbolic geometry of the Smith chart.

\section{ImPEDANCE Matching Networks}

When the impedance $Z_{m}$ required for a given phase shift is known, conventional procedures may be employed to design a matching network to match this virtual impedance to the line characteristic impedance $Z_{0}$. Smith chart techniques are frequently used to obtain circuit parameters for the matching circuits shown in Fig. 2. With the availability of electronic computing facilities, the analytic expressions for the circuit parameters are convenient and yield more precise results than the graphical methods. Expressions for two-parameter matching networks are given below.

\section{A. Quarter-Wavelength Double-Stub Transformer (Fig.} 2(a))

The impedance $Z_{m}$ to be matched may be expressed in terms of its normalized admittance $y_{m}=g_{m}+j b_{m}$ :

$$
g_{m}+j b_{m}=\frac{Z_{0}}{Z_{m}}
$$

The electrical lengths of the two stubs are 


$$
\begin{aligned}
& \theta_{1}=\mp \tan ^{-1} \sqrt{\frac{1-g_{m}}{g_{m}}} \\
& \theta_{2}=\tan ^{-1}\left[-b_{m} \mp \sqrt{g_{m}\left(1-g_{m}\right)}\right]
\end{aligned}
$$

where $\theta_{2}$ is the length of the stub nearer the termination.

\section{B. Transmission Line Transformer (Fig. 2(b))}

Characteristic impedance

$$
Z_{0 m}^{\prime}=\sqrt{Z_{0} \frac{\left(R_{m} Z_{0}-R_{m}^{2}-X_{m}^{2}\right)}{\left(Z_{0}-R_{m}\right)}}
$$

Electrical length

$$
\theta_{m}=\tan ^{-1} \sqrt{\frac{\left(Z_{0}-R_{m}\right)}{Z_{0} X_{m}^{2}}\left(R_{m} Z_{0}-R_{m}^{2}-X_{m}^{2}\right)}
$$

\section{Tandem 3/8-Wavelength Network (14) (Fig. 2(c))}

The final $\left(45^{\circ}\right)$ section of this network has a characteristic impedance equal to the magnitude of the terminating impedance

$$
Z_{m}=\sqrt{R_{m}^{2}+X_{m}^{2}}
$$

The initial $\left(90^{\circ}\right)$ section has the characteristic impedance

$$
Z_{0 m}=\sqrt{\frac{Z_{0} R_{m} Z_{m}}{\left(Z_{m}-X_{m}\right)}}
$$

\section{Transformation Network Design EXample}

The reflection-coefficient transformation method was demonstrated in a microstrip network at $1.1 \mathrm{GHz}$, using a glass-encapsulated $\mathrm{p}$-i-n diode as the semiconductor element. The diode was mounted in series connection spanning a $2.54-\mathrm{mm}$ gap in terminated $50-\Omega$ microstrip line on a $10.16-\mathrm{cm} \times 20.32-\mathrm{cm}$ substrate $3.18 \mathrm{~mm}$ thick having a relative dielectric constant of 2.32 . The diode was reverse biased with $5.0 \mathrm{~V}$, and forward-biased with a current of $2.0 \mathrm{~mA}$, in its "off" and "on" states, respectively. Impedances $Z_{1}$ and $Z_{2}$ were the impedances measured at the coaxial connector of a coaxial-to-microstrip transition to the substrate containing the diode. The observed impedances at the input to this two-state circuit at $1.1 \mathrm{GHz}$ were

$$
\begin{array}{ll}
\text { Diode ofF: } & Z_{1}=2.1+j 9.3 \Omega \\
\text { Diode oN: } & Z_{2}=33.3-j 18.3 \Omega
\end{array}
$$

These impedances correspond to reflection coefficient magnitudes of 0.92 and 0.29 , and reflection angles of $159^{\circ}$ and $-120^{\circ}$, respectively. In the transformation method, these impedances are transformed to new values which produce equal reflection amplitude and have a prescribed phase angle difference. Using the formulas given in the Appendix and specifying phase shifts of $90^{\circ}$ and $180^{\circ}$, the matching-impedance parameters of the required networks are found to be

$$
\begin{array}{ll}
\phi=90^{\circ}: & Z_{m}=9.9+j 16.4 \\
\phi=180^{\circ}: & Z_{m}=10.7+j 7.7 .
\end{array}
$$

Each calculation for $Z_{m}$ yields a pair of solutions. Solutions containing a negative real part $R_{m}$ do not represent useful matching circuit parameters. The sign of $R_{m}$ is changed by interchanging $Z_{1}$ and $Z_{2}$ (equivalent to changing the sign of $\phi$ ). Impedance transforming network forms for the diode impedance data above were selected using the criterion that circuits calling for microstrip line characteristic impedance values for which the microstrip line width is comparable to a quarter line wavelength at the selected frequency of $1.1 \mathrm{GHz}$ were avoided. Calculation with (13) to (19) showed that satisfactory microstrip line widths were obtained by the choice of a tandem twosection network (Fig. 2(c)) for the $90^{\circ}$ bit, and a singlesection line transformer (Fig. 2(b)) for the $180^{\circ}$ bit. The matching networks were constructed on $10.16-\mathrm{cm} \times 20.32$ $\mathrm{cm}$ microstrip substrates of the same material as that used for the diode mount. Dispersive microstrip line wavelength calculations were used [15], [16]. The network parameters determined for the matching networks are summarized below.

1) Impedance-Transformation Network for $90^{\circ}$ Phase Shift (Fig. 2(c)): Segment of $90^{\circ}$ electrical length: $Z_{0 m}=59$ $\Omega$, linewidth $=7.37 \mathrm{~mm}$, length $=48.51 \mathrm{~mm}$. Segment of $45^{\circ}$ electrical length: $Z_{m}=19 \Omega$, linewidth $=33.27 \mathrm{~mm}$, length $=23.40 \mathrm{~mm}$.

2) Impedance-Transformation Network for $180^{\circ}$ Phase Shift (Fig. 2(b)): Matching line segment characteristic impedance: $Z_{0 m}=21 \Omega$, width $=29.21 \mathrm{~mm}$. Electrical length of segment: $\theta_{m}=65^{\circ}$; length $=34.04 \mathrm{~mm}$. The $50-\Omega$ line width on this substrate is $9.40 \mathrm{~mm}$, and its wavelength at $1.1 \mathrm{GHz}$ is $19.46 \mathrm{~cm}$. (Dimensional tolerance approximately $0.03 \mathrm{~mm}$ ). The substrate boards containing the matching networks were connected individually to the substrate containing the diode by a coaxial line stretcher to compensate for the additional line length introduced by the coaxial connectors between boards. When diode bias was switched, the phase changes and amplitude fluctuations of the reflection coefficients were observed to be
$\phi=90^{\circ}$ with $|\Delta V|=0.2 \mathrm{~dB}$
(Nominal $90^{\circ}$ network)
$\phi=177^{\circ}$ with $|\Delta V|=0.25 \mathrm{~dB}$
(Nominal $180^{\circ}$ network)

with a measuring precision of $1^{\circ}$ and $0.1 \mathrm{~dB}$. Exact phase shifts and constancy of signal amplitude are in principle possible if impedance compensation is introduced to correct for fringing reactance in the microstrip line junctions and circuit couplings.

In the design of microstrip matching circuits, practical lower and upper bounds exist for attainable microstrip characteristic impedance. If a given two-state impedance leads to a matching requirement difficult to implement in practice, a short length of transmission line may be inserted ahead of a given two-state termination, leading to new values for $Z_{1}$ and $Z_{2}$ and a new solution for $Z_{m}$. In this way a more practicable matching condition may be reached.

In addition to the bandwidth limitation imposed by the hybrid coupler with which it is employed, a reflection phase shifter utilizing a matching network as described above is further bandwidth limited in that (4) is satisfied 
only at the single frequency of calculation. A reflectiontype phase shifter having greater bandwidth may be obtained by use of the procedure in which two-state impedance data for the semiconductor element employed is obtained by measurement over the frequency range of interest. At each frequency point measured the corresponding impedance $Z_{m}$ is calculated using the formulas in the Appendix. Standard methods for frequency-dependent impedance transformation may be then used [17], or computer-based optimization can be employed to produce the design of a broad-band matching circuit for the frequency-dependent virtual impedance $Z_{m}$.

\section{Conclusions}

A procedure has been given for the design of impedance-transforming two-port networks which transform the reflection coefficients of a switchable one-port element having the two input-impedance states $Z_{1}$ and $Z_{2}$, to new reflection coefficients having equal magnitudes and phase angles differing by a prescribed value $\phi$. Valid impedance data for $Z_{1}$ and $Z_{2}$, preferably obtained by measurement of the termination in situ, are essential to the implementation of this procedure. The method will succeed when the matching-impedance values $Z_{m}$ determined for separate phase shifts are sufficiently distinct in value that they can be transformed to the line $Z_{0}$ by attainable matching networks. This will normally be possible since new values of $Z_{m}$ for a given phase shift $\phi$ may be produced by changes in the imbedding network of the two-state element which lead to new values of the starting impedances $Z_{1}$ and $Z_{2}$.

\section{APPENDIX}

In a two-port network characterized by the voltagecurrent relations

$$
\begin{gathered}
V_{1}=A V_{2}-B I_{2} \\
I_{1}=C V_{2}-D I_{2}
\end{gathered}
$$

the matrix of general circuit parameters

$$
M=\left(\begin{array}{ll}
A & B \\
C & D
\end{array}\right)
$$

has the property that if the network is lossless and reciprocal, $A$ and $D$ are pure-real quantities, and $B$ and $C$ are pure-imaginary. The circuits are considered to be lossless and reciprocal since they will normally be composed of segments of transmission line or waveguide. Therefore, $M$ may be written as

$$
M=\left(\begin{array}{cc}
a & j b \\
j c & d
\end{array}\right)
$$

where $a, b, c, d$ are real.

The matrix of the bilinear operator $\hat{\beta}_{\Gamma} \hat{\beta}_{M}$ appearing in (12) is

$$
\begin{aligned}
\left(\hat{\beta}_{\mathrm{r}}\right)\left(\hat{\beta}_{M}\right) & =\left(\begin{array}{c}
\left(a-j c Z_{0}\right)\left(-d Z_{0}+j b\right) \\
\left(a+j c Z_{0}\right)\left(d Z_{0}+j b\right)
\end{array}\right) \\
& =\left(\begin{array}{cc}
\alpha & \beta \\
\alpha^{*} & -\beta^{*}
\end{array}\right)
\end{aligned}
$$

where $\alpha^{*}$ is the complex conjugate of $\alpha$. Equation (12) therefore is

$$
\frac{\alpha Z_{m}+\beta}{\alpha^{*} Z_{m}-\beta^{*}}=0
$$

Hence

$$
Z_{m}=-\frac{\beta}{\alpha}
$$

The network parameters are most readily determined by returning to (5). Using (A.6) in (5) leads to

$$
\frac{\alpha Z_{1}+\beta}{\alpha^{*} Z_{1}-\beta^{*}}=e^{j \phi} \frac{\alpha Z_{2}+\beta}{\alpha^{*} Z_{2}-\beta^{*}} \text {. }
$$

With (A.8), equation (A.9) becomes

$$
\frac{Z_{1}-Z_{m}}{Z_{1}+Z_{m}^{*}}=e^{j \phi} \frac{Z_{2}-Z_{m}}{Z_{2}+Z_{m}^{*}}
$$

Using $Z_{1}=R_{1}+j X_{1}$ and $Z_{2}=R_{2}+j X_{2}$ in (A.10), the real and imaginary parts separate into two equations which may be solved for $R_{m}$ and $X_{m}$, the real and imaginary components of $Z_{m}$ :

$$
\begin{aligned}
& R_{m}=-\frac{Q}{2}\left[1 \pm \sqrt{1+\frac{4 P}{Q^{2}}}\right] \\
& X_{m}=U R_{m}+V
\end{aligned}
$$

where $P, Q, U, V$ have the definitions

$$
\begin{array}{ll}
P=\frac{\left(E+M V-V^{2}\right)}{\left(1+U^{2}\right)} & E=\left(R_{1} R_{2}-X_{1} X_{2}\right) \\
Q=\frac{(K N-U M+2 U V)}{\left(1+U^{2}\right)} & M=\left(X_{1}+X_{2}\right) \\
U=K\left(\frac{R_{1}-R_{2}}{R_{1}+R_{2}}\right) & N=\left(X_{1}-X_{2}\right) \\
V=\frac{\left(R_{1} X_{2}+X_{1} R_{2}\right)}{\left(R_{1}+R_{2}\right)} & K=\left(\cot \frac{\phi}{2}\right)
\end{array}
$$

\section{ACKNOWLEDGMENT}

The author wishes to thank J. O. Taylor and R. C. Aucoin for network analyzer measurements of impedance.

\section{REFERENCES}

[1] J. F. White, "High power, p-i-n diode controlled, microwave transmission phase shifters," IEEE Trans. Microwave Theory Tech., vol. MTT-13, pp. 233-242, Mar. 1965.

[2] R. V. Garver, "Broadband diode phase shifters," IEEE Trans. Microwave Theory Tech., vol. MTT-20, pp. 314-323, May 1972.

[3] J. F. White, "Diode phase shifters for array antennas," IEEE Trans. Microwave Theory Tech., vol. MTT-22, pp. 658-674, June 1974.

[4] J. F. White, Semiconductor Control Dedham, MA Artech House, pp. 389-495.

[5] B. Glance, "A fast low loss microstrip pin phase shifter," IEEE Trans. Microwave Theory Tech., vol. MTT-27, pp. 14-16, Jan. 1979.

[6] E. Kpozdo, K. Schuenemann, and H. El-Hannawy, "A quadraphase modulator in fin line techniques," in IEEE 1979 MTT Symp. Dig., pp. 119-121, Apr. 30, 1979.

[7] K. Hirai and S. Kamihashi, "Practical design of C-band, MIC, PIN phase shifter," in IEEE 1979 Symp. Dig., pp. 229-231, Apr. 30, 1979. 
[8] P. Wahi and K. C. Gupta, "Effect of diode parameters on reflection-type phase shifters," IEEE Trans. Microwave Theory Tech, vol. MTT-24, pp. 619-621, Sept. 1976.

[9] G. Apsley, L. Coltun, and M. Rabinowitz, "Quickly devise a fast diode phase shifters," Microwaves, vol. 18, no. 5, pp. 67-68, May 1979.

[10] E. A. Guillemin, The Mathematics of Circuit Analysis. New York: Wiley 1949, pp. 363-378.

[11] H. M. Altschuler, "Matrix manipulation of bilinear transformations," Microwave J., pp. 62-65, July 1964.

[12] M. S. Navarro, "P-N junction phase modulators," M. S. thesis, Massachusetts Inst. Technol. Cambridge, Aug. 1972.

[13] D. Steinbrecher, "Circuit aspects of nonlinear microwave networks," in Proc. IEEE Int. Symp. Circuits and Systems (Boston, MA). pp. 477-479, Apr. 21, 1975.

[14] - "An interesting impedance matching network," IEEE Trans. Microwave Theory Tech., vol. MTT-15, p. 382, June 1967.

[15] W. J. Getsinger, "Microstrip dispersion model," IEEE Trans. Microwave Theory Tech., vol. MTT-21, pp. 34-39, Jan. 1973.

[16] E. Yamashita, K. Atsuki, and T. Ueda, "An approximate dispersion formula of microstrip lines for computer aided design of microwave integrated circuits," IEEE 1979 MTT Symp. Dig., pp. 320-322, Apr. 30, 1979.

[17] G. Matthaei, L. Young, and E. Jones, Microwave Filters, Impedance Matching Networks and Coupling Structures. New York: McGraw-Hill Company, 1964, p. 681.

\title{
Numerical Experiments on the Determination of Cutoff Frequencies of Waveguides of Arbitrary Cross Section
}

\author{
PATRICIO A. A. LAURA, KOSUKE NAGAYA, AND GUSTAVO SANCHEZ SARMIENTO
}

\begin{abstract}
Finding the exact mathematical solution of an electromagnetic waveguide problem is only possible in a rather limited number of technological situations. The electronic engineer usually confronts a large variety of complicating factors which makes it necessary to employ an approximate method in order to predict cutoff frequencies, propagation modes, attenuation parameters, etc.

The present paper considers only one case of complexity: a waveguide of "exotic" boundary shape.

Undoubtedly the finite-element method is the most popular technique for dealing with such a situation. Usually the accuracy of the method is tested by applying it to simple geometric domains. The purpose of the present study is twofold: first to verify its accuracy in the case of domains of complicated geometry and second to introduce an approximate approach which is based on a Fourier expansion of the boundary condition coupled with a collocation technique which may be of interest to electronic engineers.
\end{abstract}

\section{INTRODUCTION}

$\mathrm{T}$ HE use of cylindrical waveguides of complicated boundary shape is popular in many microwave engineering applications.

No attempt to present a survey of the literature will be done here since this has been accomplished in several excellent papers by Davies [1], Bates [2], Cheng Lin [3], etc.

When the boundary of the waveguide is not natural to

Manuscript received August 27, 1979; revised November 1, 1979.

P. A. A. Laura is with the Institute of Applied Mechanics, 8111 Base Naval, Puerto Belgrano, Argentina.

K. Nagaya is with the Faculty of Engineering, Yamagata University, Yonezawa, Japan.

G. Sanchez Sarmiento is with the Centro Atómico Bariloche, 8400 Rio Negro, Argentina. one of the common coordinate systems for which the governing partial differential equation can be solved by the standard method of separation of variables it is necessary to make use of approximate analytical or numerical methods.

With regards to the last ones the most general approaches are probably the finite differences and the finite elements methods. On the other hand, it is important to point out that the finite-elements approach is the most popular numerical method for the solution of problems in practically any field of applied science and engineering [4].

Most of the times the relative accuracy of the finite-element technique is demonstrated using simple configurations for which the exact solution is known.

It is the object of the present study to present a comparison of eigenvalues, in the case of TM modes, calculated: a) using the finite-element method and b) by means of two different variational approaches.

Regarding the variational approaches the following methodologies are used:

1) the cross section is transformed onto a unit circle and then Galerkin method is used to obtain an approximate solution of the governing differential system;

2) a Fourier expansion-collocation method [5].

When using the conformal mapping-variational approach one of the main difficulties of the method consists in finding the analytic function which performs the desired transformation of the given domain onto a unit 